Extensive investigations were carried out in hospital including a blood film, serum vitamin $B_{1: 2}$ and folic acid, serum electrolytes, thyroid function, E.E.G., skull radiography, and a brain scan. All these investigations gave normal results. Her spinal fluid protein concentration was $20 \mathrm{mg} / 100 \mathrm{ml}$ (normal $10-40 \mathrm{mg} / 100 \mathrm{ml}$ ). Urinary amino-acid chromatography showed normal excretions of amino-acids, but an unusual faint spot with atypical characteristics was observed. This urinary constituent has not yet been identified.

The bismuth subgaliate was stopped immediately and over a period of two weeks her condition gradually returned to normal. Her mental confusion cleared, she lost her clumsiness and tre- mulousness, and her gait became steady. She is now leading a normal life and according to her husband her intellectual functions have recovered fully.

\section{References}

Cavanagh, J. B., Greenbaum, D., Marshall, A. H. E., and Rubenstein, L. J. (1959). Lancet, 2, 524.

Corsellis, J. A. N., Goldberg, G. J., and Norton, A. R. (1968). Brain, 91, 481. Goodman, L. S., and Gilman, A. (1965). Pharmacological Basis of Therapeutics, 3rd edn. New York, Macmillan.

Heyman, A. (1944). American fournal of Syphilis, Gonorrhea and Venereal Diseases, 28, 721.

\title{
Treatment of Tetanus Neonatorum with Muscle Relaxants and Intermittent Positive-pressure Ventilation
}

\author{
P. M. SMYTHE, M. D. BOWIE, T. J. V. VOSS
}

British Medical fournal, 1974, 1, 223-226

\section{Summary}

Intermittent positive-pressure ventilation and muscle relaxants were first used in Cape Town in 1958 in an attempt to reduce the mortality from tetanus neonatorum, which was then over $90 \%$. Problems of effective ventilation, of tracheostomy, and of infection in the neonate were gradually overcome so that between 1967 and 1972 the mortality in 186 cases was $21 \%$. In a consecutive series of 97 cases the mortality was $10 \%$.

\section{Introduction}

Until preventive measures are widely established neonatal tetanus will remain a problem in many parts of the world, with a mortality in the region of $90 \%$ on conservative treatment. A regimen of treatment with intermittent positivepressure ventilation (I.P.P.V.) and muscle relaxants, which has been evolved after much trial and error, is described because it has stood the test of time and of changes of staff, has greatly reduced the mortality and, it is hoped, will prove helpful in areas where tetanus neonatorum is still a problem.

\section{Patients and Methods}

On admission to hospital all cases received 1-2 $\mathrm{ml}$ paraldehyde intramuscularly or 1-2 mg diazepam (Valium) to help control the spasms and were given oxygen as required. Antitetanic serum 10-20,000 $U$ intravenously and 20,000 $U$ intramuscularly in divided doses was given at four different sites on the outer aspect of the thigh. Intramuscular gammaglobulin 2 $\mathrm{ml}$ helped to control infection, and $1-2 \mathrm{mg}$ vitamin $K_{1}$ was

University of Natal, Durban, Natal, South Africa

P. M. SMYTHE, M.D., F.R.C.P., Professor of Paediatrics and Child Health

University of Cape Town, Cape, South Africa

M. D. BOWIE, F.R.C.P., D.C.H., Senior Lecturer, Department of Paediatrics and Child Health; Principal Paediatrician, Red Cross War Memorial Children's Hospital, Rondebosch, Cape

T. J. V. VOSS, M.B., D.A., Senior Lecturer, Department of Anaesthesia; Principal Anaesthetist, Red Cross War Memorial Children's Hospital, Rondebosch, Cape

given intramuscularly to minimize bleeding at tracheostomy. Intramuscular procaine penicillin $100,000 \mathrm{U}$ and kanamycin $15 \mathrm{mg} / \mathrm{kg} /$ day in two doses were given for 10 days to control the unbilical infection and supply antibiotic cover.

A small percentage of neonates with tetanus survived on a conservative regimen of tube feeding and sedation. These infants had mild spasms which did not materially interfere with breathing or swallowing saliva. Severe spasms, a severe cyanotic episode, or any apnoeic episode were absolute indications for tracheostomy and assisted respiration.

\section{TRACHEOSTOMY}

Tracheostomy is best carried out under general anaesthesia with the use of an endotracheal tube. Local anaesthesia alone increases the risk of the procedure. A high tracheostomy with a longitudinal incision through the second, third, and fourth tracheal rings using a short incision of exposure has virtually abolished the complication of pneumothorax. Excision of any cartilage or a transverse incision of the trachea was found to be undesirable.

A Pilling-Holinger tracheostomy tube $5 \mathrm{~mm}$ in diameter (size 1) and $33 \mathrm{~mm}$ long had a T-piece welded on to the inlet to provide a side attachment to the respirator and an end opening for suctioning (fig. 1). Care was taken to see that the spiggot blocking the opening at the end was firmly fixed and could not blow out. Without any cuff the tube fitted snugly into the lumen of the trachea preventing any appreciable leak

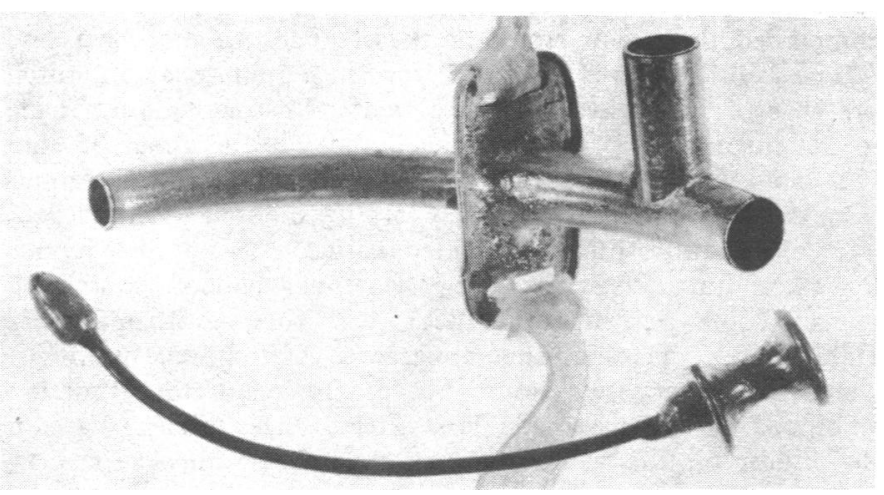

FIG. 1-Pilling-Holinger tracheostomy tube showing T-piece welded on for attachment to ventilator. 
of air when positive pressure was applied. Pressure erosions of the infant's trachea developed very easily, and to lessen the chances of this occurring the tapes holding the tracheostomy tube were not tied too tightly and the tube was kept loose enough to be pulled in and out for $0.5 \mathrm{~cm}$ each day so that it did not apply constant pressure on one site. The attachment to the respirator was light and flexible (biliary $T$ tubing) and the tube was not so long as to impinge on the carina. The angle of the head, neck, and body were such as to ensure that the tracheostomy tube and the tracheal lumen were in alignment.

\section{VENTILATION}

The best ventilator was found to be the one with which the nursing and medical staff were most familiar, and the simpler the better. The East Radcliff ventilator working through a warm water humidifier proved excellent. The humidifier was set so that the gases entering the patient were between 33$35^{\circ} \mathrm{C}$. This meant that the humidifier was set at $\pm 60^{\circ} \mathrm{C}$. Good thermostatic control of the humidifier was essential, as failure of the thermostat might have meant death of the child. Tubing was angled to allow all water condensation to drain back into the humidifier. More modern systems are available but satisfactory results were obtained with the simple system shown in fig. 2 .

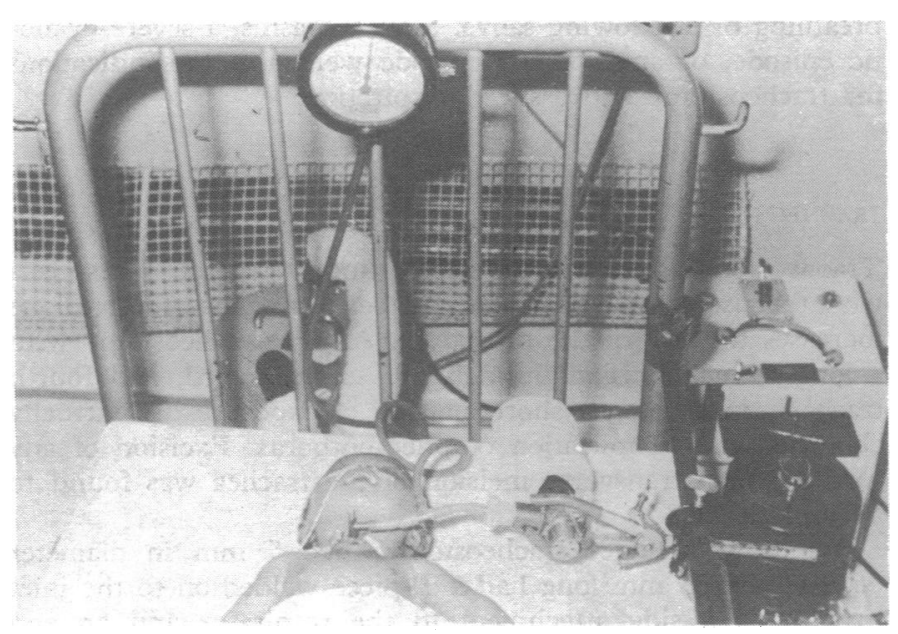

FIG. 2-A simple system used for ventilating most infants.

At the time of tracheostomy all equipment was ready, with the humidifier warmed, for attachment of the infant to the ventilator with a minimum of delay to prevent drying and inspissation of tracheal secretions. A medical officer always accompanied the infant from the theatre back to the ward.

The ventilator was run at 37 cycles a minute at an initial pressure of $15 \mathrm{~cm}$ water. Subsequent adjustments were made to the inspiratory pressure to keep the $\mathrm{PCO}_{2}$ at about $32 \mathrm{~mm}$ Hg. If an Astrup apparatus is not available the mixed venous $\mathrm{PCO}_{2}$ can be measured by a rebreathing method (Sykes, 1960) by which equilibrium is obtained between the mixed venous $\mathrm{PCO}_{2}$, the lung gas, and a bag or rubber balloon containing oxygen. The percentage of $\mathrm{CO}_{2}$ was then estimated in a Haldane $\mathrm{CO}_{2}$ gas analysing apparatus. At first measurements were made every two hours but as the respiratory function stabilized they were made less of ten. Once efficient care of the infant on the ventilator was established checks of $\mathrm{PcO}_{2}$ were rarely required and many infants were successfully treated without any check at all. Accurate recordings, by showing a rise in pulse rate, gave warnings of impairment of respiratory function. Most infants were successfully ventilated using air only, which had the advantage of cyanosis appearing early as a manifestation of inadequate ventilation. Recently more sophisticated studies have shown presence of subclinical hypoxia, and $30-40 \%$ oxygen in air is preferable to air. Higher inspiratory percentages of oxygen lead to respirator lung syndrome and diffuse atelectasis. Therefore inspired oxygen should not exceed $30-40 \%$ unless specific indications exist. An Ambu bag, or some other means of hand ventilation, must always be available in case of ventilator failure.

\section{MUSCULAR RELAXATION}

Muscular relaxation was maintained with $10 \mathrm{mg}$ of tubocurarine chloride given intramuscularly. Injections were repeated whenever muscular twitchings recurred. Some recovery of muscular action was allowed between each injection of tubocurarine otherwise ileus might have developed. Infants were nursed on their backs for the duration of I.P.P.V. to avoid displacement of the tracheostomy tube. Nursing the infant with his head inclined upwards by about $15^{\circ}$ helped to drain the upper lobe bronchi, the upper lobes being most liable to collapse. Severe flattening of the occiput and pressure sores could have developed if the head had not been turned every half-hour. This was best achieved by fitting a tray under the mattress on which the whole child and the ventilator attachment was tipped to one side or the other without disturbing the attachment of tracheostomy tube to respirator. Some suppont under the neck took weight off the head but was only enough to keep the tracheostomy tube in correct alignment with the trachea. A foam rubber mattress shaped to the contour of the infant's neck and back distributed pressure evenly.

\section{HAZARDS}

Maintenance of a clear airway was the most important factor in obtaining good results. Any deterioration in the child's condition focused attention on airway obstruction. Obstructions sometimes occurred in the tubing leading to the child; the insertion of a manometer attached to the $T$-piece biliary tubing both helped in localization of an obstruction and provided the nurses with an easily visible indicator of the functioning of the ventilator. Another danger was inspissation of mucus which could obstruct the tracheostomy tube, particularly if humidification was inadequate. Also incorrect alignment of the tracheostomy tube could tilt the tube so that the end impinged on the tracheal wall-an indication of this having occurred was difficulty in passing the suctioning catheter. Most often obstruction followed accumulation of secretions in the trachea and bronchi, especially in the smaller bronchi. Aspiration was carried out hourly and later every two hours. Using a sterile No. 3 English gauge or No. 8 French gauge rubber catheter with a whistle tip (end and side openings) reduced the risk of adherence to the tracheal mucous membrane. If a whistle tip catheter is not available then a catheter with its tip cut off obliquely should be used. Strict sterile precautions were taken during suctioning and catheters were rinsed through with sterile water which was discarded after use. Saliva accumulated in quantity in the mouth of the curarized child. It was sucked out often, especially before suctioning the trachea and bronchi as it might have leaked past the tracheostomy tube down into the air passages.

Physiotherapy was carried out every four hours to bring up secretions obstructing the smaller bronchi. Only practice made this efficient; hyperinflation of the lung with positive pressure was followed by compression of the chest wall with both hands 
to simulate a cough. Pressure on the diaphragm was applied by the thumbs pressing upwards under the costal margin. Too vigorous compression can result in fractured ribs. So long as rhonchi could be felt or auscultated, or there was diminished air-entry or movement of any area of the chest, physiotherapy and suctioning was continued. $X$-ray pictures were sometimes needed to show the collapse or the re-expansion of a collapsed lobe immediately after efficient physiotherapy.

Infection was the other great hazard to these infants. If there was infection in the stoma around the tracheostomy tube, the tube was changed daily (but not during the first four days), otherwise weekly. The importance of preventing pressure by the tracheostomy tube on the tracheal wall must again be stressed, as an ulcer at the site may act as a nidus of infection, and the need to prevent stagnation of secretions in the bronchi and collapse of segments of lung is also important. Systemic antibiotics may help, but a dramatic reduction of infection resulted from the instillation of $0.25 \mathrm{ml}$ of sterile solution of penicillin $500 \mathrm{U}$, and colistin $500 \mathrm{U}$ (Smythe, 1967), freshly made up each day, down the tracheostomy tube every four hours for the first two days. Afterwards this treatment was continued only if the secretions became purulent. Bacteriological identification and drug sensitivity of the infecting organism may indicate a change of antibiotics.

\section{FEEDING}

Feeding was by gastric tube. Half-strength Darrow's solution was given for the first 48 hours. The stomach was aspirated before each feed; any residue indicated some ileus. Milk feeds were not started until there was no residue. Ileus could have occurred at any time and the abdomen was palpated daily for faeces in the left iliac fossa. If these were felt clear feeds were given only until the bowels acted. Occasionally a quarter of a bisacodyl (Dulcolax) suppository was required.

\section{WEANING FROM VENTILATION}

After a period of between two and four weeks tetanic muscular twitching lessened and the period between injections of tubocurarine lengthened. Spontaneous respiratory movements occurred at this time and a trial was made of stopping curare. Phenobarbitone $7.5 \mathrm{mg}$ was given three times a day by stomach tube to maintain relaxation. The ventilator had to be continued for four to five days after stopping curare, however, gradually reducing the inspiratory pressure, as sudden fatal apnoea can occur for no apparent reason during the period of re-establishment of normal breathing; as if the respiratory centre becomes unresponsive after prolonged artificial take over.

After respiration had been re-established, the hypertonicity of the jaw muscles relaxed sufficiently for sucking movements to be made and feeding by mouth was attempted with a Bellcroy feeder. Only when this was carried out without any difficulty was an attempt made to detubate.

A size 00 Pilling-Holinger tracheostomy tube of $3 \mathrm{~mm}$ diameter was inserted and the stoma of the tube blocked with the bung from a stomach tube. The diameter of the trachea was so much larger that breathing around the tube through the nose or mouth usually resulted. When the tube could be occluded continuously for 24 hours and all feeds were taken without difficulty the tube could be removed without likelihood of its having to be reinserted.

If the infant would not tolerate blocking of the tabe this indicated that one of three types of obstruction was present (Smythe, 1964); granulations which had formed above the level of the tracheostomy tube; compression of the tracheal rings above the level of the stoma; or angling or kinking of the trachea with inspiration because it was attached by fibrous tissue forming around the stoma to the skin. The methods that were used to effect detubation in these children are described elsewhere (Smythe, 1967). Severe ulceration of the tracheal wall could result in a stricture developing.

Immunization with tetanus toxoid was started before the infant was discharged as the amount of toxin which causes the disease is inadequate to produce a state of immunity.

Tetanus is a preventable disease and neonatal tetanus should never occur with good obstetrical practice. Nevertheless, where this is not available and the risk is high the disease can still be prevented by immunization of the pregnant mother with tetanus toxoid.

All the tubing was thoroughly washed out before sterilization. Boiling water or steam was used for the humidifier and only sterile water was added when in use. Ventilators were best sterilized using ethylene dioxide but if this is not available it does not mean that this method of therapy should be abandoned. The risk of infection is low if the ventilator is used only for this type of patient. On one occasion three infants developed tuberculosis which was later traced to a ventilator having been used on a child with miliary tuberculosis.

\section{Results and Discussion}

Altogether 415 children have been treated for tetanus neonatorum, of whom 267 survived. The mortality of over $90 \%$ in 1956-7 fell to $21 \%$ in 186 cases admitted between 1967 and 1972 (fig. 3). I.P.P.V. was first tried in 1959 (Smythe and Bull, 1959) with some improvement, but it was the introduction of the Sykes rebreathing method (Sykes, 1960) to control the inspiratory pressure which caused a sharp reduction in mortality after 1961 (Smythe, 1963). The deterioration in 1964 was largely a result of problems with tracheostomy; when these were clarified (Smythe, 1964) improvement followed. In 1967 a further sharp fall in mortality followed the introduction of the instillation of antibiotics down the tracheostomy tube to control infection (Smythe, 1967). Since then the only significant rise in mortality was in 1970 when for three weeks $100 \%$ oxygen was used in error (fig. 3). But for this the mortality would have been $16 \%$ in 155 cases. In a consecutive series of 97 admissions there were 10 deaths. In the last 30 admissions there have been no deaths.

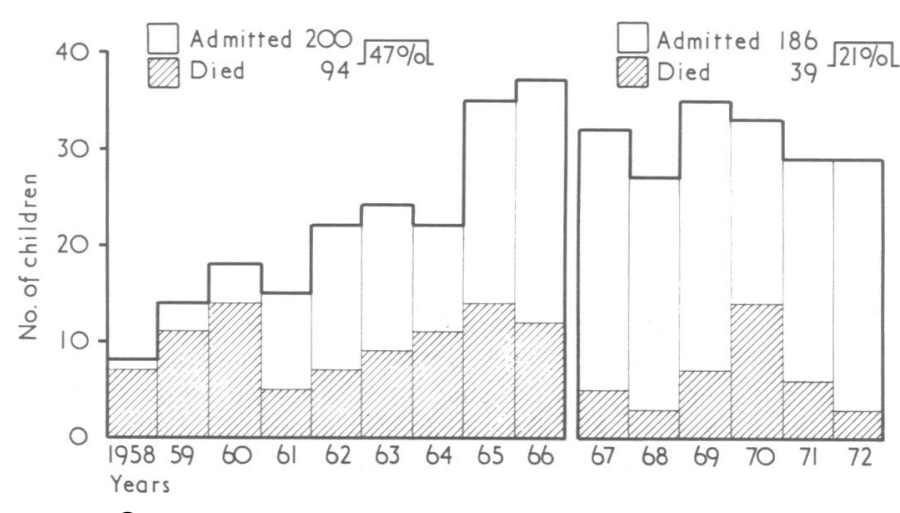

FIG. 3-Change in mortality rate after solving problems of I.V.P.P. by 1966. Rise in 1970 was due to an error in oxygen administration.

It is not our purpose to compare and contrast different methods and results. But it should be stressed that other methods have been tried, and deviations from the recommended procedures should be approached with caution.

This unit has been under the care of doctors who have other responsibilities as well as the tetanus unit. About 30 infants are 
treated each year in a unit of six beds. Generally at any one time two infants are on curare and I.P.P.V., two are being weaned from the ventilator, and two are in the process of being detubated. The infants stay in the wand from six to eight weeks.

the single most important contributory factor towards success in treatment is the constant care and attention by the doctor in charge. Until the method is perfected and the nursing staff are familiar with the hazards, the doctor must visit the child every four hours. If he cannot sleep in he must see the child at midnight and again first thing in the morning. The doctor must make himself fully familiar with the techniques, particularly of physiotherapy and suctioning, after which assistants and registrars can be trained to take over part of the care. The same applies to the nursing staff, who must be trained in sufficient numbers to provide full 24 -hour cover. It is useless to try to treat these children if an untrained nurse is expected to take over the care of a child who can die in five minutes should anything go wrong. Nevertheless, high praise is due to the nursing staff who are not trained nurses, but nurseaides; care and attention and dedication has been largely responsible for the good results.
ADDENDUM.- Recently a positive end expiratory pressure of $5 \mathrm{~cm}$ water has been used with ventilation of these infants (Voss, 1973). At this early stage the results seem promising, with the absence of atelectasis of the upper lobes, reduction in respiratory infection, and a great improvement in the shape of the chest. This expiratory pressure is easily obtained by letting the end of the expiratory tube open under $5 \mathrm{~cm}$ of water.

We thank the ear, nose, and throat surgeons, especially $\mathrm{Mr}$. J. Marais, for doing the tracheostomies, and the medical superintendent of the Red Cross War Memorial Children's Hospital for permission to publish.

Requests for reprints should be addressed to: Dr. M. D. Bowie, Department of Paediatrics, Medical School, University of Cape Town, Mowbray, Cape, South Africa.

\section{References}

Smythe, P. M. (1967). Lancet, 1, 335.

Smythe, P. M. (1964). Fournal of Pediatrics, 65, 446.

Smythe, P. M., and Bull, A. (1959). British Medical fournal, 2, 107.

Smythe, P. M. (1963). British Medical fournal, 1, 565.

Sykes, M. K. (1960). British fournal of Anaesthesia, 32, 256.

Voss, T. J. V. (1973). South African Medical Fournal, 47, 761.

\section{Can I Have an Ambulance, Doctor?}

T. C. BEER,

E. GOLDENBERG,

D. S. SMITH,

British Medical fournal, 1974, 1, 226-228

\section{Summary}

In a study of the ambulance service to an outpatient physiotherapy centre in an urban area over half of the palients were aged over 65, a quarter made at least three visits to the centre weekly, and the average travel and waiting times for all patients was about two-and-a-half hours. There was a direct relation between distance from home to the centre and travelling time for morning but not afternoon attenders. A second survey after some changes in the transport arrangements showed some relatively small reductions in overall travelling and waiting time. It is concluded that even under favourable conditions an important side effect of outpatient physiotherapy is the probable fatigue and anxiety generated by ambulance travel.

\section{Introduction}

The study by the Organisation and Method Group of the Department of Health ${ }^{1}$ on outpatient departments and the ambulance service showed that over half of all outpatient ambulance journeys were made by patients attending physiotherapy departments. The report suggested that any test of the efficiency of the transport service for outpatients must therefore be concentrated on the arrangements for patients attending these departments and other frequent ambulance users such as fracture clinics.

Northwick Park Hospital, Harrow, Middlesex HA1 3UJ

T. C. BEER, M.B., M.R.C.P., Senior Registrar

E. GOLDENBERG, S.R.N., Research Assistant

D. S. SMITH, D.PHYS.MED., F.R.C.P., Consultant Physician

A. STUART MASON, M.B., M.R.C.P., Member of scientific staff, M.R.C and D.H.S.S. Epidemiology and Medical Care Unit

\section{A. STUART MASON}

In view of this and because of the increasing emphasis on rehabilitation services we have studied the transport arrangements for patients attending the Harrow Physical Treatment Centre, which is in effect the outpatient physiotherapy department of Northwick Park Hospital.

\section{Organization}

Some 800 attendances are made at the centre each week. Twelve per cent. of the patients are brought by ambulance; the hospital car service is not used for this purpose.

Outpatient physiotherapy is ordered by a hospital doctor working in one of the hospitals in the Northwick Park group. There is no direct general-practitioner access to physiotherapy services. When physiotherapy is ordered a note is made on whether ambulance transport will be required. This information is passed to the hospital transport officer, who orders an ambulance to take the patient to the centre for the first day of treatment. Subsequently transport is ordered by a clerk at the centre, who sends a block booking to the ambulance service for the duration of the course of treatment, as ordered by the doctor.

Transport is cancelled only by a doctor, who does not ordinarily review the patient until the treatment has been completed. Thus even if the patient recovers sufficiently during treatment to make his own way to the centre the ambulance will continue to call. Contact between centre and ambulance control occurs only when a new patient has to be "block booked" or a patient has had to cancel his treatment: it does not take place every day.

Ninety five per cent. of patients walk well enough to be collected in a single-handed, eight-seater ambulance which during the day is confined to serving the centre. Each evening the driver receives from ambulance control a list of the patients he is to pick up next day. He does three or four rounds a day depending on how many patients require treatment and their place of residence.

Patients are told that the ambulance will call sometime after 8.30 a.m. (all morning attenders) or after 1.30 p.m. (all afternoon 